\title{
Two-dimensional phononic band structure of archimedean-logarithmic spiral-based slabs
}

\author{
Selami Palaz ${ }^{a}$, Oral Oltulu ${ }^{a}$, Amirullah M. Mamedov ${ }^{\mathrm{b}, \mathrm{c}}$, and Ekmel Ozbay ${ }^{\mathrm{b}}$ \\ ${ }^{a}$ Department of Physics, Faculty of Sciences, Harran University, Sanliurfa, Turkey; ${ }^{b}$ Nanotechnology \\ Research Center (NANOTAM), Bilkent University, Bilkent, Ankara, Turkey; ${ }^{C}$ International Scientific Center, \\ Baku State University, Baku, Azerbaijan
}

\begin{abstract}
We present band structure results for elastic waves in periodic composite materials consisting of a spiral scatterer shape embedded in a uniform silicon matrix. The material of the scatterer is tungsten as a high density material. The phononic band structure of two-dimensional solid phononic crystal is studied numerically by finite element method to obtain dispersion relations. We find full band gaps at relatively low frequencies for a low filling ratio. Due to spatial inhomogeneity, the unique structural characteristics of the spiral structure lead to localized modes. Hence, the proposed model geometry introduces a phononic crystal to cover a wide range of stopbands starting from low frequencies. The results could give a possibility to design effective filters for the low frequency range.
\end{abstract}

\section{ARTICLE HISTORY}

Received 25 June 2018

Accepted 23 December 2018

\section{KEYWORDS}

Elastic waves; phononic crystals; metamaterials; band gaps

\section{Introduction}

The study of wave propagation in periodic structures consisting of at least two different materials has been a subject of intense research in physics. These periodic composites known as phononic crystals (PCs) have been utilized by their property of generating full band gaps, or frequency ranges where propagation of elastic or acoustic waves through crystal is forbidden for any crystalline direction [1-5]. Phononic crystals are generally defined by artificial materials or engineered materials, which are also known as metamaterials $[6,7]$.

The material features, such as elastic constants, mass densities, shape, size and/or spatial arrangement of the scatterers strongly affect the wave propagation in the structure [8]. Periodic arrangement of scatterers embedded in a host matrix with these properties can give absolute band gaps. The band gaps can be tuned by changing the mentioned features in the lattice [9-11]. However, tuning of a bandgap by changing the crystal and material parameters is a difficult task in practice.

The existence of full band gaps has been extensively investigated both theoretically and experimentally [12-14]. Phononic crystals with low-frequency band gaps may lead to applications in sound isolation, as well as seismic wave shielding, low-frequency waveguides and ultrasonics [15]. This type of phononic crystals may have the possibility 
of tailoring the dispersion properties and the frequency ranges of complete band gaps of elastic waves for functional control of wave propagation. From a fundamental viewpoint, low-frequency forbidden bands of elastic waves in 2D PCs are needed in the field of Micro-electromechanical Systems (MEMS) [16].

In last few years, the possibility of phonon confinements in a set of spirally connected phononic crystals are investigated numerically and experimentally [17-20]. In this work, we focus on a solid-solid PC to investigate a broad bandgap at low frequencies. We demonstrate the utility of a spiral-shaped phononic crystal.

Spirals are ubiquitous and found extensively in nature, ranging from the spiral structure of galaxies to banded patterns in rocks to the earth, humans, animals, plants and insects. Since nature is abundant in spirals, such as seashells, horns, and bones, we suggest a new PC by modeling a spiral-shaped structure. The modal behavior was utilized to understand the elastic wave propagation in a confined spiral configuration. Spatial filtering of frequencies through the system was studied. In the following, we proposed a two-dimensional square phononic crystal made of two materials, silicon and tungsten. These materials create a strong contrast between both their mechanical properties such as the elastic constants, as well as their densities, meaning that silicon can be considered as the soft material while tungsten is the hard one. The contrast of the material properties between the two structures is particularly important to band gap generation.

\section{Materials and method}

Since the spiral pattern turns out to be omnipresent in our natural world, this led us to present a different spiral model. The most popular spirals are the spiral of Archimedes and the logarithmic spiral. The Archimedean spiral is a shape that paces outward from a point called center as it revolves around the central point. On the other hand, the logarithmic spiral is one of the simplest spirals for which the radius grows exponentially with the angle and gains in speed as it moves away from its center. Therefore, a new spiral pattern can be constructed in a different manner from the combination of both Archimedean and logarithmic spirals. We can describe the new spiral defined by a mathematical function with the following equation in polar coordinates [21]:

$$
r=(a+b \theta) e^{i \theta}
$$

where $r$ is the distance from the origin, $a$ and $b$ are arbitrary constants that define the initial radius of the spiral and the spiral growth rate. The angle $\theta$ is the angle from the $x$-axis. The spiral equation can be converted from polar to Cartesian coordinate system by multiplying with $f(\theta)$, where the function $f(\theta)$ is $\cos (\theta)$ and $\sin (\theta)$ for $\mathrm{x}$ and y components in the Cartesian coordinates, respectively.

The considered system in this study is 2D phononic crystal composed of spiralshaped Tungsten inclusions in silicon background arranged in a square lattice. The calculation model of the studied system and its unit cell are shown in Figure 1.

In the absence of an external force, the equation of motion for inhomogenous solids the wave equation is [22]: 


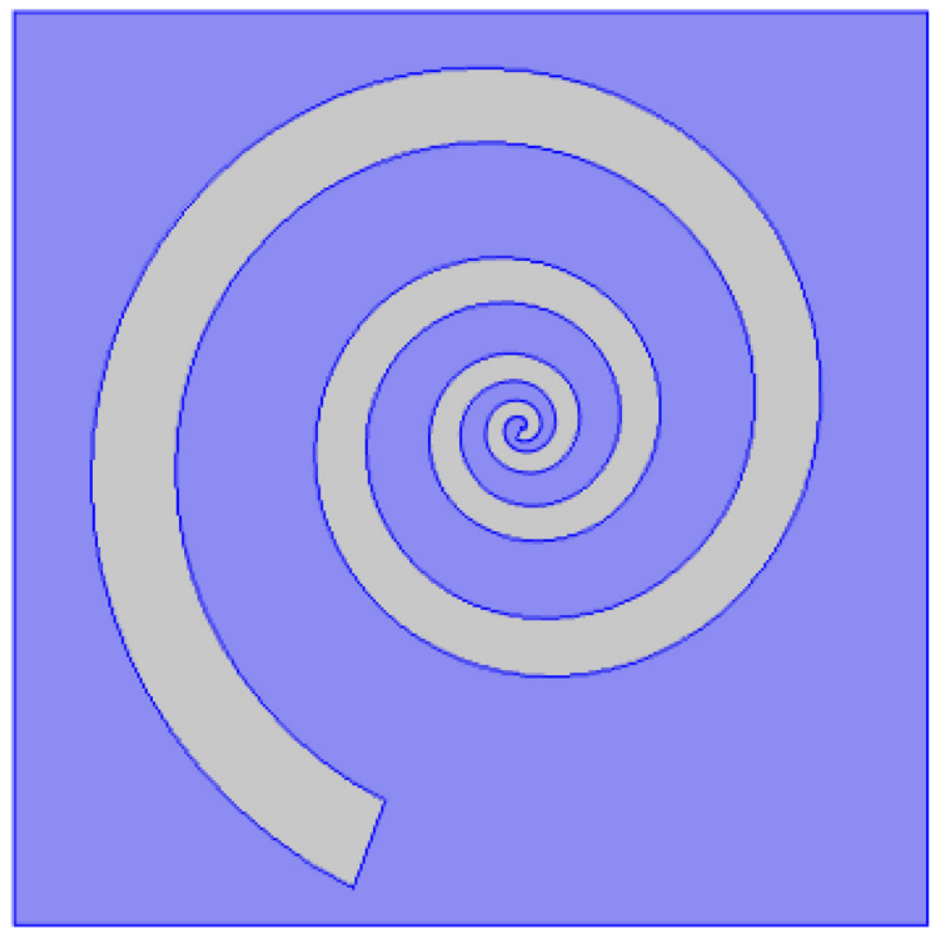

Figure 1. A two dimensional cross section of the spiral-based periodic structure arranged on a square lattice.

$$
\frac{\partial^{2} u_{j}^{i}}{\partial t^{2}}=\frac{1}{\rho_{j}}\left\{\frac{\partial}{\partial x_{i}}\left(\lambda \frac{\partial u_{j}^{i}}{\partial x_{l}}\right)+\frac{\partial}{\partial x_{l}}\left[\mu\left(\frac{\partial u_{j}^{i}}{\partial x_{l}}+\frac{\partial u_{j}^{l}}{\partial x_{i}}\right)\right]\right\}
$$

where $\mathrm{u}^{\mathrm{i}}$ is the $i$-th component of the displacement vector $(\mathrm{i}=1,2,3)$ in the Cartesian coordinate system $\left(\mathrm{x}_{1}, \mathrm{x}_{2}, \mathrm{x}_{3}\right), \lambda(\vec{r})$ and $\mu(\vec{r})$ are the Lame coefficients, and $\rho(\vec{r})$ is the density, and the subscript $j$ refers to the medium. The Lame coefficients can be expressed in terms of the longitudinal and transverse speed of sound and they are given by Young's modulus $E$.

$$
\begin{gathered}
E_{t}=\rho c_{t}^{2}=\mu, \\
E_{l}=\rho c_{l}^{2}=\lambda+2 \mu,
\end{gathered}
$$

Assuming the structure is homogeneous along the $\mathrm{x}_{3}$, the wave propagation is reduced to two dimensions in $\left(\mathrm{x}_{1}, \mathrm{x}_{2}\right)$ plane. Oscillations polarized in $\left(\mathrm{x}_{1}, \mathrm{x}_{2}\right)$ planes are known as $\mathrm{XY}$ modes. Due to its importance, only the $X Y$ mode will be considered here. According to the Bloch theorem, the elastic displacement can be written in the following form:

$$
\vec{u}(\vec{r})=\vec{u}_{\vec{k}}(\vec{r}) e^{i \vec{k} \cdot \vec{r}}
$$

where $\boldsymbol{k}=\left(k_{\mathrm{x}}, k_{\mathrm{y}}\right)$ is the wave vector limited to the first Brillouin zone of the repeated lattice. Due to the periodicity of the structure, the calculation can be performed on a representative unit cell. The discrete form of the eigenvalue equations in the unit cell can be written as 

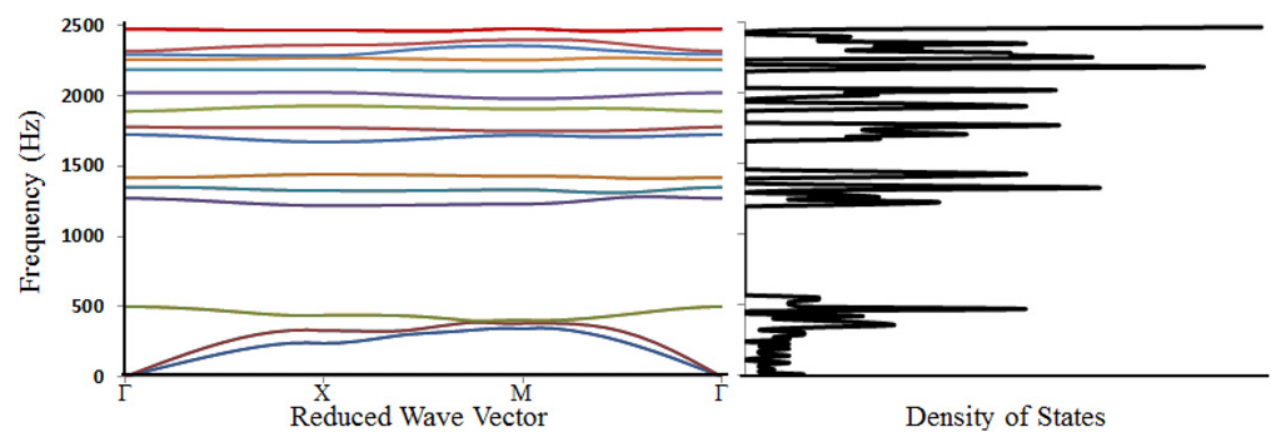

Figure 2. Elastic band diagram of infinite PnCs composed of spiral-based array of tungsten inclusions in silicon matrix. The lattice constant is a $=40 \mathrm{~mm}$. Density of states (DOS) calculated for the 2D phononic crystal system.

$$
\left(K-\omega^{2} M\right) U=0
$$

where $U$ is the displacements at the nodes; $K$ and $M$ can be considered as stiffness and mass matrices of the unit cell, respectively. With the Bloch-Floquet theorem, Bloch periodic boundary conditions were applied on the boundaries of the unit cell:

$$
\vec{U}(\vec{r}+\vec{a})=\vec{U}_{\vec{k}}(\vec{r}) e^{i \vec{k} \cdot \vec{a}} .
$$

The eigenvalue equation associated with $\mathrm{XY}$ mode was solved with finite element modeling using Comsol Multiphysics ${ }^{\circledR}$ software under the periodic boundary condition.

\section{Numerical results and discussions}

Figure 2a shows the calculated band structure of a square array of spiral shaped tungsten inclusions in silicon matrix, where the lattice constant $a$ is $40 \mathrm{~mm}$. The Young's modulus, Poisson ratio and density of the silicon matrix are $\mathrm{E}=4.1 \times 10^{6} \mathrm{GPa}$, $\nu=0.021$, and $\rho=1280 \mathrm{~kg} / \mathrm{m}^{3}$ as a background material. The Young's modulus, Poisson ratio and density of the tungsten material are $\mathrm{E}=3.6 \times 10^{11} \mathrm{~Pa}, \nu=0.27$, and $\rho=17800 \mathrm{~kg} / \mathrm{m}^{3}$, respectively.

The inset in Figure 2a shows the first Brillouin zone and high symmetry points. It is apparent in the calculated band structure that nine wave modes are contained in the frequency range from 0 to $2.5 \mathrm{kHz}$. Within the nine modes, absolute bandgaps are clearly seen in Figure 3. The first gap locates between the third and fourth mode and ranges from $0.49 \mathrm{kHz}$ to $1.21 \mathrm{kHz}$, with a gap width of $0.72 \mathrm{k} \mathrm{Hz}$. The second gap is located between the fourth and fifth band, ranging from $1.28 \mathrm{kHz}$ to $1.31 \mathrm{kHz}$. The band gap width is $0.03 \mathrm{kHz}$. The third gap is located between the fifth and sixth band, ranging from $1.41 \mathrm{kHz}$ to $1.35 \mathrm{kHz}$. The band gap width is $0.06 \mathrm{kHz}$. The fourth gap is located between the sixth and seventh band, ranging from $1.44 \mathrm{kHz}$ to $1.67 \mathrm{kHz}$. The band gap width is $0.23 \mathrm{kHz}$. The fifth gap is located between the eighth and ninth band, ranging from $1.72 \mathrm{kHz}$ to $1.74 \mathrm{kHz}$. The band gap width is $0.02 \mathrm{kHz}$. The sixth gap is located between the eight and ninth band, ranging from $1.77 \mathrm{kHz}$ to $1.88 \mathrm{kHz}$. The band gap width is $0.11 \mathrm{kHz}$. The seventh gap is located between the ninth and tenth band, ranging from $1.92 \mathrm{kHz}$ to $1.97 \mathrm{kHz}$. The band gap width is $0.05 \mathrm{kHz}$. The eighth gap is 


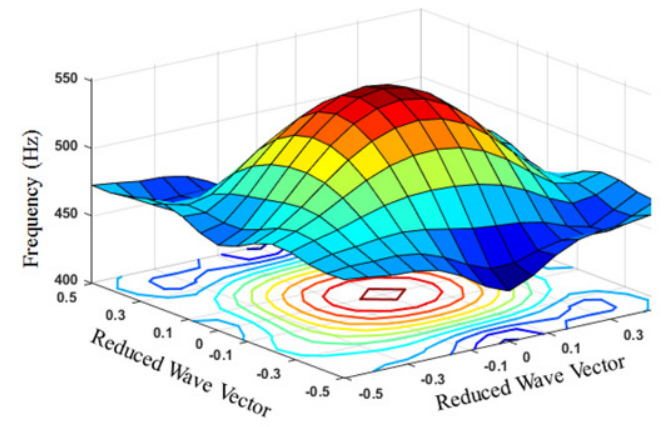

a

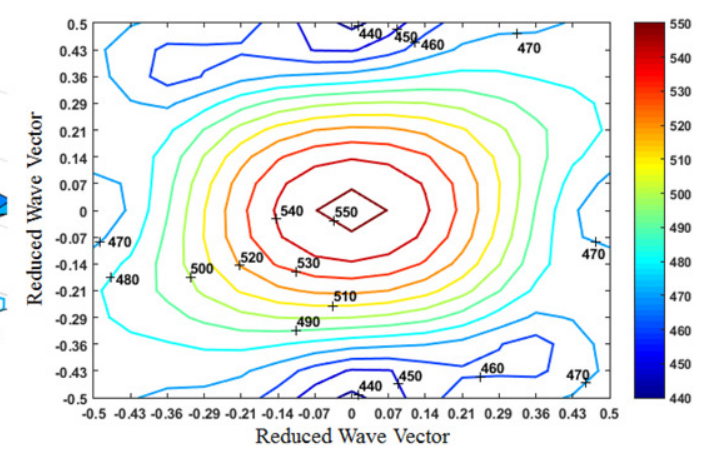

b

Figure 3. The dispersion, $\omega(\mathrm{k})$, relation (a) and the equi-frequency contours for the third mode (b) of the square PC, calculated for all k-vectors in the first Brillouin zone.

located between the tenth and eleventh band, ranging from $2.02 \mathrm{kHz}$ to $2.17 \mathrm{kHz}$. The band gap width is $0.15 \mathrm{kHz}$. The ninth gap is located between the eleventh and twelfth band, ranging from $2.39 \mathrm{kHz}$ to $2.45 \mathrm{kHz}$. The band gap width is $0.06 \mathrm{kHz}$. It is evident that significant band gaps are available at low frequencies. The appearance of these absolute bands at low frequencies is due to the complexity of the medium.

DOS calculation was carried out by sampling k-points in the two dimensional Brillouin Zone. A sum of all bands over all frequencies yields the density of states as a function of frequency. DOS calculations are particularly important since some physical properties, such as thermal conductivity and specific heat, can be modified by changing the phonon density of states in a phononic crystal. They provide insight into both microscopic and macroscopic properties. Figure $2 \mathrm{~b}$ shows the calculated DOS states as a function of frequency. The location and gap width of these gaps are corresponding well with the result in Figure $2 \mathrm{a}$ and $\mathrm{b}$, validating the numerical results of the band structure calculations.

The refraction as well as reflection characteristics of the composite can be best understood by introducing a three-dimensional frequency-graph. The corresponding frequencygraphs are called the dispersion surfaces of the elastic waves, as are shown in Figures 3a and $4 \mathrm{a}$. The dispersion surfaces are also known as equi-frequency surfaces (EFSs) and they are all related to the eigen modes contained inside the unit cell. The EFS can be formed by calculating all the allowed Eigen modes by varying the $k$ vectors inside the entire first Brillouin zone. Each point through the entire surface is a result of the possible eigen solutions that consist of all the allowed wave vectors in the first Brillouin zone.

To understand the isotropic or anisotropic propagation of elastic waves in PC, the equi-frequency contours (EFCs), were calculated at selected frequencies. By definition, the contours of constant frequency along the $k_{x}$ and $k_{y}$ wave-vector plane are called equi-frequency contours (EFCs). The contour shapes are non-circular in Figures $3 \mathrm{~b}$ and $4 \mathrm{~b}$, which mean anisotropic phase velocity of the elastic waves. Therefore, the non-circular contours indicate different directions of phase and energy velocities. Near the $\mathrm{X}$ point, the allowed wave vector changes a curvature, and becomes virtually a circle. Hence, this contour shows a negative refraction effect.

Figure $4 \mathrm{~b}$ shows that the EFCs are far from being a circle for the entire fourth band, indicating a strong anisotropy. Since the gradient of the frequency $\omega$ with respect to the 


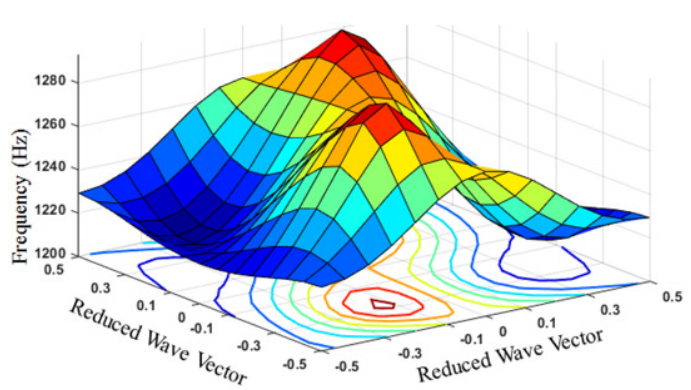

a

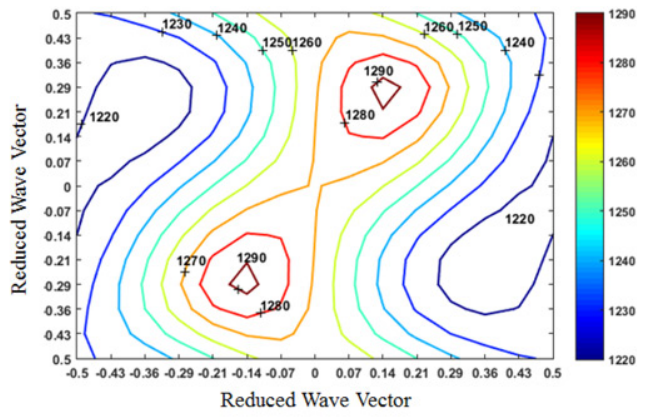

b

Figure 4. The dispersion, $\omega(\mathrm{k})$, relation (a) and the equi-frequency contours for the fourth mode (b) of the square $\mathrm{PC}$, calculated for all k-vectors in the first Brillouin zone.

wave vector $\mathrm{k}$ is the group velocity, the orientation of group velocity vectors is always perpendicular to the EFCs. Therefore, for a range of incident wave vectors, the energy of the modes will propagate with group velocity, and it is aligned in the increasing $\omega(\mathrm{k})$ direction. Elastic waves propagate with diffraction in the curved regions of the EFC. In Figure $4 \mathrm{~b}$ the allowed wave vector shifts into a different shape, and the center is not located on the $\Gamma$ point. That is, there is a change in the curvature near the $\mathrm{X}$ and $\mathrm{M}$ points. The reverse change in curvature is due to inhomogeneous medium. When the contour shows a change in curvature, the self-collimation, prism and lens effects can be observed. For nearly flat regions in the figure, self-collimation effect takes place.

\section{Conclusion}

We considered a new anisotropic design of solid elastic metamaterials to study wave propagation. A series of numerical simulation for the full waveforms of elastic waves was performed in the frequency domain in order to investigate the band gap characteristics of the proposed PC structure. The modal behavior was utilized to understand the elastic wave propagation in a confined spiral configuration. Spatial filtering of frequencies through the system was found. It is found that significant band gaps are available at low frequencies. The appearance of these absolute bands at low frequencies is due to the complexity of the medium. It must be noted that the filling ratio for the composite system is small. For such a small filling ratio, noticeable pass-bands were obtained at low frequencies.

\section{Acknowledgement}

One of the authors, Ekmel Ozbay, acknowledges partial support from the Turkish Academy of Sciences.

\section{References}

[1] M. S. Kushwaha et al., Acoustic band structure of periodic elastic composites, Phys. Rev. Lett. 71 (13), 2022 (1993). DOI: 10.1103/PhysRevLett.71.2022.

[2] M. S. Kushwaha, and P. Halevi, Band-gap engineering in periodic elastic composites, Appl. Phys. Lett. 64 (9), 1085 (1994). DOI: 10.1063/1.110940. 
[3] P. Lambin et al., Stopping of acoustic waves by sonic polymer-fluid composites, Phys. Rev. E Stat. Nonlin. Soft. Matter. Phys. 63 (6 Pt 2), 066605 (2001). DOI: 10.1103/ PhysRevE.63.066605.

[4] J. O. Vasseur et al., Absolute forbidden bands and waveguiding in two-dimensional phononic crystal plates, Phys. Rev. B. 77, 085415 (2008).

[5] Y. Pennec et al., Phononic crystals and manipulation of sound, Phys. Status Solidi. 6 (9), 2080 (2009). DOI: 10.1002/pssc.200881760.

[6] M.-H. Lu, L. Feng, and Y.-F. Chen, Phononic crystals and acoustic metamaterials, Mater. Today. 12 (12), 34 (2009). DOI: 10.1016/S1369-7021(09)70315-3.

[7] X. Zhou, X. Liu, and G. Hu, Elastic metamaterials with local resonances: an overview, Theor. Appl. Mech. Lett. 2 (4), 041001 (2012). DOI: 10.1063/2.1204101.

[8] R. Lucklum, Phononic crystals and metamaterials - promising new sensor platforms, Procedia Eng. 87, 40 (2014). DOI: 10.1016/j.proeng.2014.11.261.

[9] M. Sigalas, and E. Economou, Elastic and acoustic wave band structure, J. Sound Vib. 158 (2), 377 (1992). DOI: 10.1016/0022-460X(92)90059-7.

[10] M. Sigalas, and E. Economou, Band structure of elastic waves in two dimensional systems, Solid State Commun. 86 (3), 141 (1993). DOI: 10.1016/0038-1098(93)90888-T.

[11] Y. Yao, Z. Hou, and Y. Liu, The two-dimensional phononic band gaps tuned by the position of the additional rod, Phys. Lett. A. 362 (5-6), 494 (2007). DOI: 10.1016/ j.physleta.2006.10.061.

[12] Z. Liu et al., Elastic wave scattering by periodic structures of spherical objects: Theory and experiment, Phys. Rev. B. 62 (4), 2446 (2000). DOI: 10.1103/PhysRevB.62.2446.

[13] Y. Tanaka, Y. Tomoyasu, and S. Tamura, Band structure of acoustic waves in phononic lattices: Two-dimensional composites with large acoustic mismatch, Phys. Rev. B. 62 (11), 7387 (2000). DOI: 10.1103/PhysRevB.62.7387.

[14] Y. Pennec et al., Two-dimensional phononic crystals: examples and applications, Surf. Sci. Rep. 65 (8), 229 (2010). DOI: 10.1016/j.surfrep.2010.08.002.

[15] Z. Liu et al., Locally resonant sonic materials, Science. 289 (5485), 1734 (2000).

[16] N. Wang et al., Micromechanical resonators based on silicon two-dimensional phononic crystals of square lattice, J. Microelectromech. Syst. 21 (4), 801 (2012). DOI: 10.1109/ JMEMS.2011.2174416.

[17] S. Zhang, J. H. Wua, and Z. Hu, Low-frequency locally resonant band-gaps in phononic crystal plates with periodic spiral resonators, J. Appl. Phys. 113 (16), 163511 (2013). DOI: $10.1063 / 1.4803075$.

[18] A. Shelke et al., Spiral lamb waveguide for spatial filtration of frequencies in a confined space, Exp. Mech. 55(7), 1199 (2015). DOI: 10.1007/s11340-015-0018-0.

[19] D. Simard, Y. Painchaud, and S. LaRochelle, Integrated Bragg gratings in spiral waveguides, Opt. Express. 21 (7), 8953 (2013). DOI: 10.1364/OE.21.008953.

[20] S. Banerjee, and R. U. Ahmed, Phonon confinement using spirally designed elastic resonators in discrete continuum, IJMSA. 3 (1), 6 (2014). DOI: 10.11648/j.ijmsa.20140301.12.

[21] https://www.comsol.com/blogs/how-to-build-a-parameterized-archimedean-spiralgeometry.

[22] E. A. Rietman, and J. M. Glynn, Band-gap engineering of phononic crystals: a computational survey of two-dimensional systems, Physics. 1, 12 (2007). http://arxiv.org/abs/0708. 3669v1. 